\title{
Hubble diagram of gamma-ray bursts calibrated with Gurzadyan-Xue cosmology
}

\author{
H. J. Mosquera Cuesta ${ }^{1}$, R. Turcati ${ }^{1}$, C. Furlanetto ${ }^{1}$, H. G. Khachatryan ${ }^{2}$, S. Mirzoyan ${ }^{2}$, and G. Yegorian ${ }^{2}$ \\ 1 Instituto de Cosmologia, Relatividade e Astrofísica(ICRA-BR), Centro Brasileiro de Pesquisas Físicas Rua Dr. Xavier Sigaud 150, \\ CEP 22290-180, Urca, Rio de Janeiro, RJ, Brazil \\ e-mail: hermanjc@cbpf.br \\ 2 Yerevan State University and Yerevan Physics Institute, Yerevan, Armenia
}

Received 8 July 2007 / Accepted 21 April 2008

\begin{abstract}
Context. Because gamma-ray bursts (GRBs) are the most luminous among known cosmic objects, they carry an essential potential for cosmological studies if properly used as standard candles.

Aims. We used GRBs to test the cosmological predictions of the Gurzadyan-Xue (GX) model of dark energy, a novel theory that predicts, without any free parameters, the current vacuum fluctuation energy density close to the value inferred from the SNIa observations. We also compared the GX results with those predicted by the concordance scenario $\Lambda$-CDM.

Methods. After a consistent calibration for a specific model, using the several empirical relations obtained from GRBs observables enables one to probe current cosmological models. Based on this recently introduced method, we used several samples from different sources that fit our needs, including 69 GRBs, the recently released SWIFT satellite data, and 41 GRBs that have firmly determined redshifts. These data samples span a distance scale up to redshift about 7.

Results. We show that the GX models are compatible with the Hubble diagram of the 69 GRBs sample. Such an adjustment is almost identical to the one for the concordance $\Lambda$-CDM. From this particular analysis, we can obtain the corresponding values of the matter density parameter $\Omega_{\mathrm{m}}$ describing GX models. When the same procedure is applied to the SWIFT satellite data, we verify that the SWIFT sample does not delineate a Hubble diagram as clearly as featured by the 69 GRBs sample.

Conclusions. The analysis of these 3 samples shows that more data and effort are needed to elucidate both issues: the gamma-ray bursts/standard-candle and the lack of a theoretical understanding of the physics that may explain the empirical relations.
\end{abstract}

Key words. gamma rays: bursts - cosmology: miscellaneous

\section{Introduction}

Gamma-ray bursts (GRBs) have been used for cosmography aims, for analysing the high-redshift behavior of the $\Lambda$-CDM cosmological scenario, as well as for several alternative cosmologies (Schaefer 2003, 2007; Bloom et al. 2003; Dai et al. 2004; Ghirlanda et al. 2004; Friedman \& Bloom 2005; Liang \& Zhang 2005, 2006; Xu et al. 2005; Wang \& Dai 2006; Firmani et al. 2006). The potential power of GRBs for cosmological studies, obviously, resides both in their very high luminosity, which is the highest among known astrophysical objects, and in the fact that they undergo practically no extinction over cosmological distances. It is therefore possible to trace significantly higher distance scales than is possible via supernovae, that is, to trace very deep into the expansion history of the universe. The situation, however, is not that simple.

Schaefer (2007) has developed a statistical approach based on the empirical correlations obtained from several observed GRBs characteristics and obtained the Hubble diagram for the GRBs after calibrating them for the concordance and other cosmological models. This approach has also been used in Mosquera Cuesta et al. (2006a,b, 2008). The key issue is the use of the empirical relations, e.g. by Ghirlanda, Liang-Zhang and others, in the absence of understanding their underlying nature, i.e., the genuine character of the scatter in the GRBs luminosity vs. luminosity indicators relations and of their mutual links. To this difficulty Schaefer (2007) has shown that, although the scatter of each of the empirical relations cannot be that small, their joint action can lead to smaller scatter, useful for probing certain cosmological models. Increase in the statistics and deeper studies of the systematics and selection effects will certainly increase the usefulness of Schaefer's approach.

In the present paper we use the same approach as Schaefer (2007) to obtain the GRB Hubble diagram for the cosmological models proposed by Gurzadyan \& Xue (2002, 2003). The original motivation for GX cosmological models is that they predict the current vacuum fluctuation energy density close to the value inferred from the SNIa observations (Perlmutter et al. 1998; Riess et al. 1998; Perlmutter et al. 1999; Riess et al. 2004, 2006) without any free parameter.

\section{The cosmological models}

Gurzadyan \& Xue $(2002,2003)$ have derived a formula for the dark energy that fits the observed value without free parameters:

$$
\rho_{\mathrm{GX}}=\frac{\pi}{8} \frac{\hbar c}{L_{\mathrm{p}}^{2}} \frac{1}{a^{2}}=\frac{\pi}{8} \frac{c^{4}}{G} \frac{1}{a^{2}},
$$

where $\hbar$ is the Planck constant, the Planck length is $L_{\mathrm{p}}=$ $(\hbar G)^{\frac{1}{2}} c^{-3 / 2}, c$ the speed of light, and $G$ the gravitational constant. Here $a$ is the upper cutoff scale in computation of vacuum fluctuations, and it has to be close to the event horizon 
(Djorgovski \& Gurzadyan 2007). According to Zeldovich (1967), the vacuum energy (1) corresponds to the cosmological term. The GX formula (1) defines a broad set of cosmological models (Vereshchagin 2006). For it the existence of a separatrix was shown Vereshchagin \& Yegorian (2006a), which divides the space of cosmological solutions into two classes: Friedmannianlike with initial singularity and non-Friedmannian solutions that begin with nonzero scale factor and vanishing matter density. Each solution is characterized by the single quantity, a density parameter that is defined in the same way as in the standard cosmological model $\Omega_{\mathrm{m}}=\frac{8 \pi G_{0} \mu_{0}}{3 H_{0}^{2}}$, where $\mu$ is the matter density, $H$ the Hubble parameter, and index " 0 " refers to their values today. The separatrix is given by

$\Omega_{\mathrm{sep}}=\frac{2}{3} \frac{1}{1-\frac{K}{\pi^{2}}} \approx \frac{2}{3}$,

where $K= \pm 1,0$ parametrize the spatial curvature. The origin of the separatrix was revealed in Khachatryan (2007), and attributed the invariants in GX models.

Analytical solutions for the GX models both for matter density and the scale factor have been obtained (Vereshchagin \& Yegorian 2006b). It turns out that the most simple solutions for the scale factor are again those of the separatrix. In one model it is exponential, in the others they are polynomials. Vereshchagin \& Yegorian (2006b, 2008) generalized GX models to include radiation and looked for other consequences of the models.

The predictions of the GX models were shown to be compatible with supernovae and cosmic microwave background data in Djorgovski \& Gurzadyan (2007). A likelihood analysis of supernovae and radio galaxies data was performed in Vereshchagin \& Yegorian (2006c) and Khachatryan et al. (2007).

\section{Luminosity distance formula for GX-models}

The models are described by the two equations for the mass density and scale factor (Vereshchagin \& Yegorian 2006b)

$$
\begin{aligned}
\dot{\mu}+3 H\left(\mu+\frac{p}{c^{2}}\right) & =-\dot{\mu}_{\Lambda}+\left(\mu+\mu_{\Lambda}\right)\left(\frac{2 \dot{c}}{c}-\frac{\dot{G}}{G}\right), \\
H^{2}+\frac{k c^{2}}{a^{2}}-\frac{\Lambda}{3} & =\frac{8 \pi G}{3} \mu,
\end{aligned}
$$

where a dot denotes the time derivative. For matter (pressure $p=0$ ), we have a solution for matter mass density with GX-dark energy (Khachatryan 2007)

$\mu_{\mathrm{m}}(t)=\left(b_{\mathrm{m}}^{\mathrm{GX}}+\frac{\pi a(t)}{4}\right) \frac{c^{2}(t)}{G(t) a^{3}(t)}$,

where $b_{\mathrm{m}}$ is a GX-invariant for matter. For the scale factor,

$\dot{a}(t)=c(t) \sqrt{\frac{8 \pi b_{\mathrm{m}}^{\mathrm{GX}}}{3 a(t)}+\pi^{2}-k}$.

The luminosity distance $d_{\mathrm{L}}$ is (Peebles 1993; Daly \& Djorgovski 2005)

$$
\begin{aligned}
d_{\mathrm{L}}(z) & =a_{0} f_{k}\left(\kappa_{\mathrm{S}}\right)(1+z), \\
\kappa_{\mathrm{S}} & =\frac{1}{a_{0} H_{0}} \int_{0}^{z} \frac{c(\xi)}{h(z)} \mathrm{d} z, \\
h(z) & =\frac{H(z)}{H_{0}}, 1+z=\frac{a_{0}}{a}
\end{aligned}
$$

where $\kappa_{\mathrm{S}}, z$ are normalized distance and redshift, respectively. The function $f_{k}\left(\kappa_{\mathrm{s}}\right)$ is defined as

$$
f_{k}(x)=\left\{\begin{array}{c}
\sin (x), k=1 \\
x, k=0 \\
\sinh (x), k=-1
\end{array}\right.
$$

where $k$ is the effective curvature $K-\pi^{2} / 3$ as in (2). The luminosity distance $d_{\mathrm{L}}(z)$ for GX models

$d_{\mathrm{L}}(z)=a_{0}(1+z) f_{k}\left(\frac{1}{\sqrt{\beta}} \ln \left|\frac{g(z)-1}{g(0)-1} \frac{g(0)+1}{g(z)+1}\right|\right)$,

where

$g(z)=\sqrt{\frac{\alpha}{\beta}(z+1)+1}, \quad \alpha=\frac{8 \pi b_{\mathrm{m}}^{\mathrm{GX}}}{3 a_{0}}, \quad \beta=\pi^{2}-k$.

For the separatrix $\alpha=b_{\mathrm{m}}^{\mathrm{GX}}=0$, we have a simple equation for the luminosity distance

$d_{\mathrm{L}}(z)=a_{0}(1+z) f_{k}\left(\frac{\ln |z+1|}{\sqrt{\pi^{2}-k}}\right)$

\section{Gamma-ray bursts calibrated with GX models}

For the present analysis we benefit of the largest GRB sample having properly determined the currently available redshifts and luminosities. The first was constructed by Schaefer (2007) and includes 69 GRBs whose main observables are time lag, variability, peak energy, maximum energy in $\gamma$-rays, and rise time, which were obtained from the GRBs data provided by many $\gamma$-ray and X-ray satellites and ground-based observatories, as collected in Greiner's homepage: http: //www .mpe.mpg.de/ $\sim j c g / g r b g e n . h t m l$. The second one is the 41 GRBs sample with firmly determined redshift as collected by Rizzuto et al. (2007) from the SWIFT satellite.

We performed the calibration procedure (linear regression analysis) of five luminosity relations: time lag vs. luminosity $\left(\tau_{\text {lag }}-L\right)$, variability vs. luminosity $(V-L)$, peak energy vs. luminosity $\left(E_{\text {peak }}-L\right)$, peak energy vs. geometrically corrected gamma-ray energy $\left(E_{\text {peak }}-E_{\gamma}\right)$, and risetime vs. luminosity $\left(\tau_{\mathrm{RT}}-L\right)$ (Schaefer 2007; Mosquera Cuesta et al. 2006a,b, 2008). We used the OLS Bisector method (Isobe et al. 1990) to find a relation between each pair of these GRBs observational properties. The best-fit line for all luminosity relations is given by the general expression $\log \mathcal{L}=a+b \log \mathcal{I}$, where $\mathcal{L}$ is the luminosity, $\mathcal{I}$ the luminosity indicator, $a$ the intercept, and $b$ the slope in each of the calibration plots presented here. Their uncertainties are

$\sigma_{\log \left(L_{i}\right)}^{2}=\sigma_{a}^{2}+\left(\sigma_{b} x_{i}\right)^{2}+\left(b \sigma_{x_{i}}\right)^{2}+\sigma_{\mathrm{sys}}^{2}$,

where $\sigma_{\log L_{i}}$ defines the standard deviation in the luminosity $L_{i}$, $\sigma_{a}$ the standard deviation in the intercept $a, \sigma_{b}$ is the standard deviation in the slope $b, \sigma_{x_{i}}$ the standard deviation in each $x_{i}$ variable representing $\log I$, and $\sigma_{\text {sys }}$ the systematic error associated to each luminosity $L_{i}$ estimate. The results of the calibration procedure are given in the Table 1 , and the plot for all luminosity relations is given in Fig. 1. 


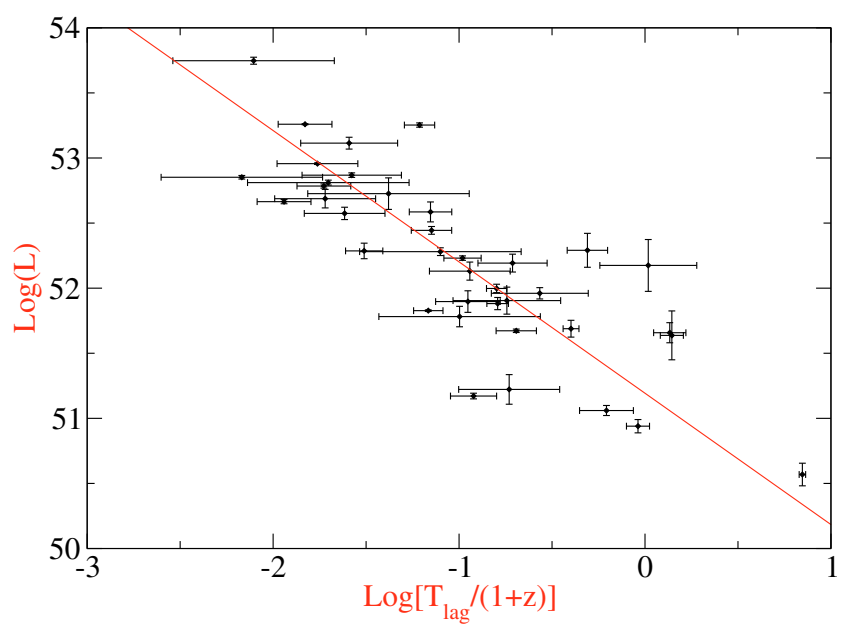

(a) Time lag vs. $L$

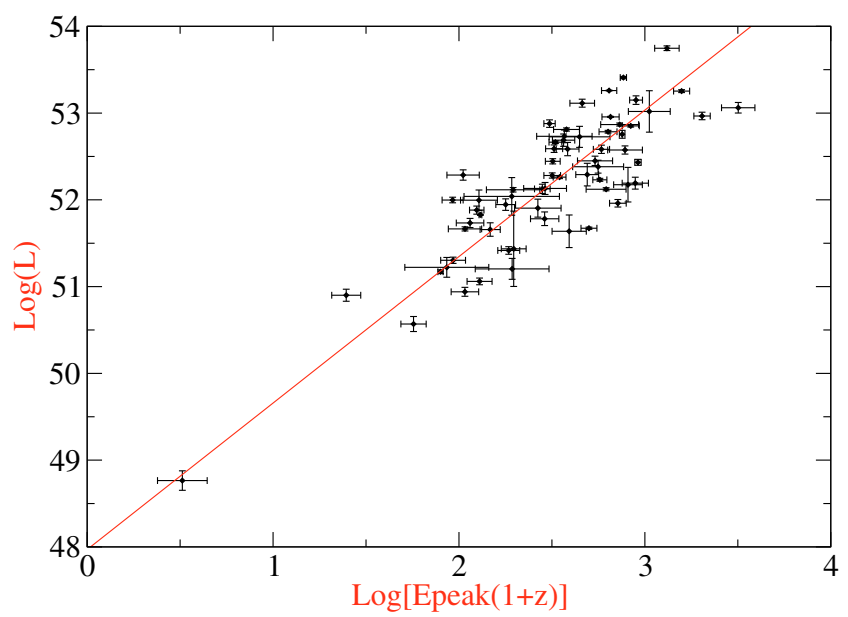

(c) $E_{\text {peak }}$ vs. $L$

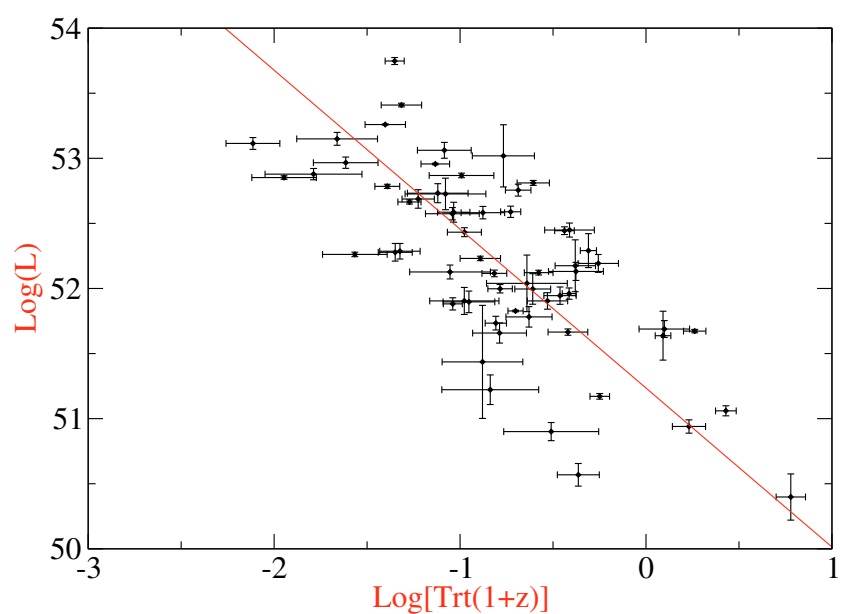

(e) Rise time vs. $L$

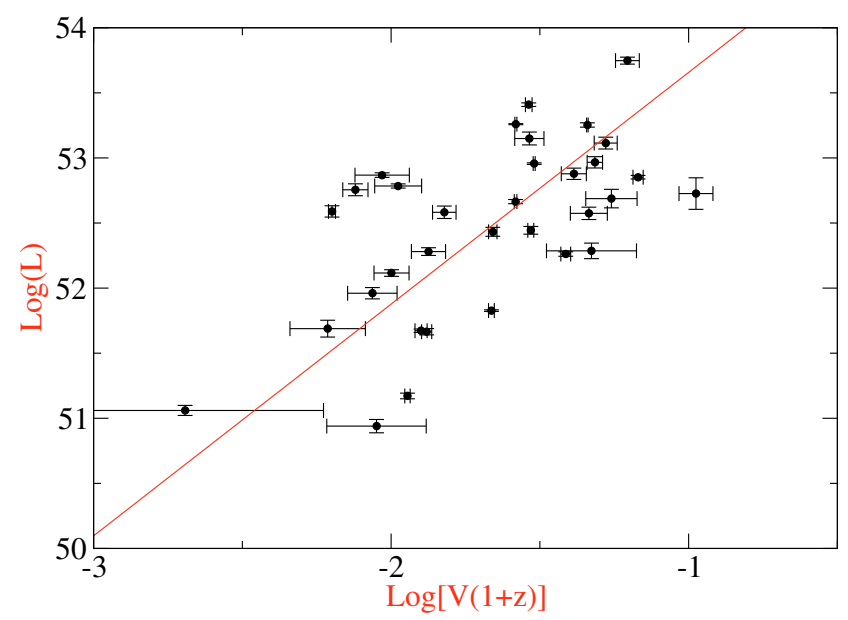

(b) Variability vs. $L$

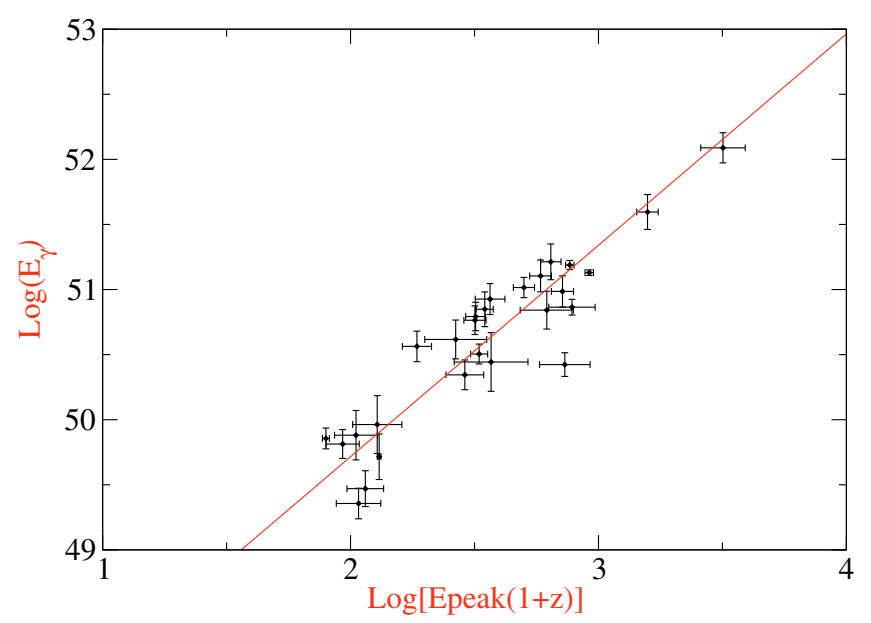

(d) $E_{\text {peak }}$ vs. $\mathrm{E}_{\gamma}$ (Ghirlanda Relation)

Fig. 1. (Color online) Results of the calibration procedure. All relations were corrected to the restframe of the GRB and also used the luminosity best-fit line obtained from the nonlinear regression method. a) Time lags for 39 GRBs. b) Variability for 51 GRBs vs. isotropic luminosity. c) $E_{\text {peak }}$ values for 64 GRBs vs. isotropic luminosity. d) $E_{\text {peak }}$ values for 27 GRBs vs. total burst energy in the gamma rays. e) Rise time for 62 GRBs vs. isotropic luminosity. 


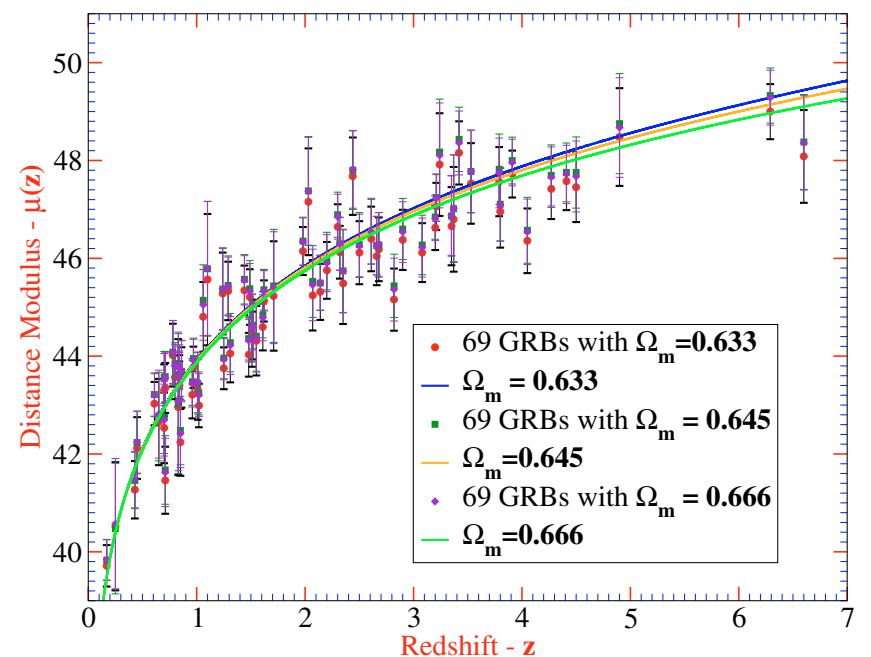

(a) $\mathrm{k}=0$

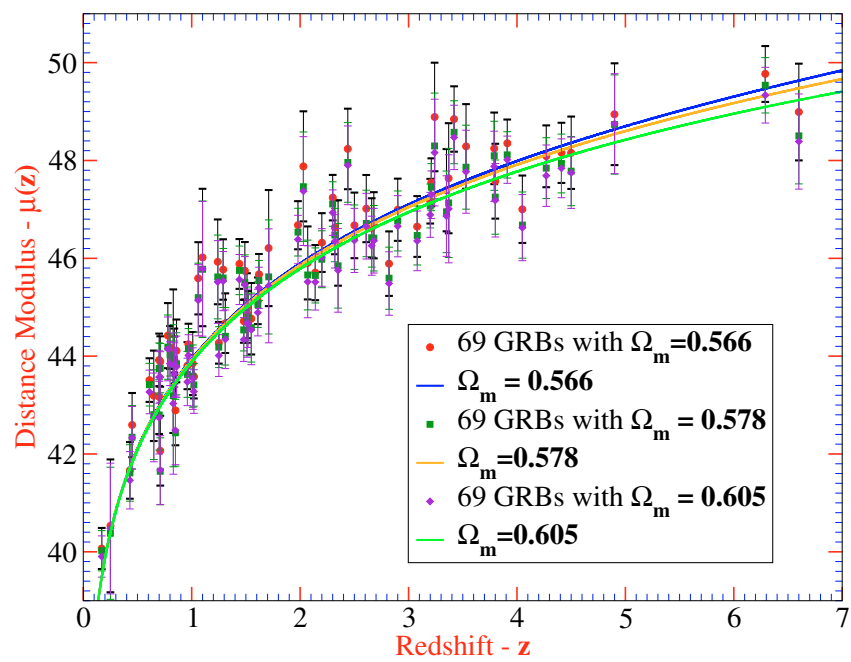

(b) $\mathrm{k}=-1$

Fig. 2. (Color online) Hubble diagram of 69 GRBs calibrated with GX models. a) Case $k=0$ and different values of $\Omega_{\mathrm{m}}$. b) Case $k=-1$ and different values of $\Omega_{\mathrm{m}}$. The corresponding parameters are indicated in the insets.

Table 1. Calibration results.

\begin{tabular}{lccccc}
\hline \hline Luminosity relation & $a$ & $\sigma_{a}$ & $b$ & $\sigma_{b}$ & $\sigma_{\text {sys }}$ \\
\hline$\tau_{\text {lag }}-L$ & 52.20 & 0.07 & -1.01 & 0.09 & 0.36 \\
$V-L$ & 52.41 & 0.08 & 1.78 & 0.19 & 0.47 \\
$E_{\text {peak }}-L$ & 52.15 & 0.05 & 1.69 & 0.10 & 0.41 \\
$E_{\text {peak }}-E_{\gamma}$ & 50.49 & 0.05 & 1.62 & 0.11 & 0.21 \\
$\tau_{\mathrm{RT}}-L$ & 52.45 & 0.07 & -1.22 & 0.11 & 0.47 \\
\hline
\end{tabular}

Using the same method as Schaefer (2007), we obtained the best estimated distance moduli $\bar{\mu}_{i}{ }^{1}$

$\bar{\mu}=\frac{1}{w} \sum_{i=1}^{5} w_{i} \bar{\mu}_{i}, \quad w_{i}=1 / \sigma_{i}^{2} \quad$ and $\quad\left[w=\sum_{i=1}^{5} w_{i}\right]$,

where the summation is over the relations with available data, $\bar{\mu}_{i}$ is the best estimated distance modulus from the $i$ th relation, and $\sigma_{i}$ the corresponding uncertainty. Then applying the error propagation law to the Eq. (13) we obtained the standard deviation associated to this best estimated as $\sigma_{\bar{\mu}}=1 / \sqrt{w}$.

Figure 3 presents the Hubble Diagram for the 69 GRBs calibrated with the GX models and its comparison with the $\Lambda$-CDM model for the parameters given in the inset. Figure 2 shows the Hubble Diagram for the 69 GRBs calibrated with the GX models obtained after a slight variation in the separatrix solution for each cosmological model, i.e., depending on the curvature $k=0,-1$.

\footnotetext{
${ }^{1}$ For an outlying source of apparent, $m$, and absolute, $M$, magnitudes, distance estimates are made through the distance-modulus: $\bar{\mu} \equiv m-$ $M$, which is related to the luminosity distance $d_{\mathrm{L}}$ (given below in units of $\mathrm{Mpc}$ )
}

$d_{\mathrm{L}}=a_{0}(1+z) \int_{a}^{a_{0}} \frac{\mathrm{d} a}{a \dot{a}}$,

through the expression

$\bar{\mu}(z) \equiv m-M=5 \log _{10} d_{\mathrm{L}}(z)+25$.

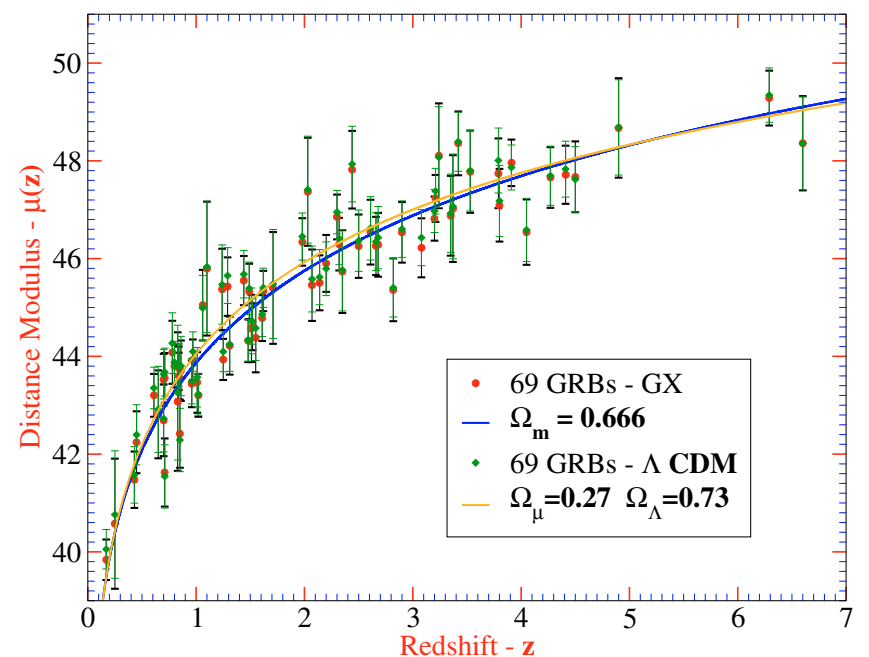

Fig. 3. (Color online) Hubble diagram of 69 GRBs calibrated with GX models as obtained from the empirical relations provided by Fig. 1. The blue line represents GX models calibrated with $\Omega_{\mathrm{m}}=0.66, k=0$, and $H_{0}=70 \mathrm{~km} \mathrm{~s}^{-1} \mathrm{Mpc}^{-1}$. Orange line is for the concordance cosmology $\Omega_{\mathrm{m}}=0.27, \Omega_{\Lambda}=0.73$.

\section{Results}

\subsection{GX compatibility with the 69 GRBs sample}

We compared GX models with the data set of 69 GRBs in Schaefer (2007). We used a general least square technique for all models with different density and curvature parameters. We find the best fit as $\Omega_{\mathrm{m}}=2 / 3, k=0$. The $\chi^{2}$ value for those parameters is 1.037 with 68 DoF. The best fit curve is shown in Fig. 4a for the value of the curvature parameter $k=0$, while in Fig. $4 \mathrm{~b}$ the best fit curve is shown for $k=-1$. In both figures, which combine GRBs, supernova type Ia, and radio-galaxies, several other HD from the GX model are plotted for different parameters $\Omega_{\mathrm{m}}$, as indicated. 


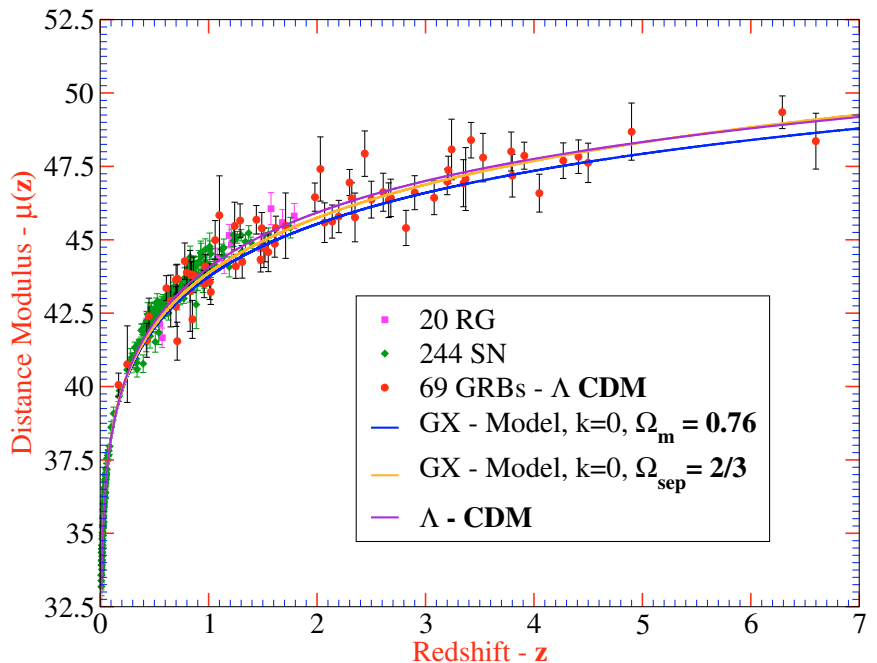

(a) $\mathrm{k}=0$

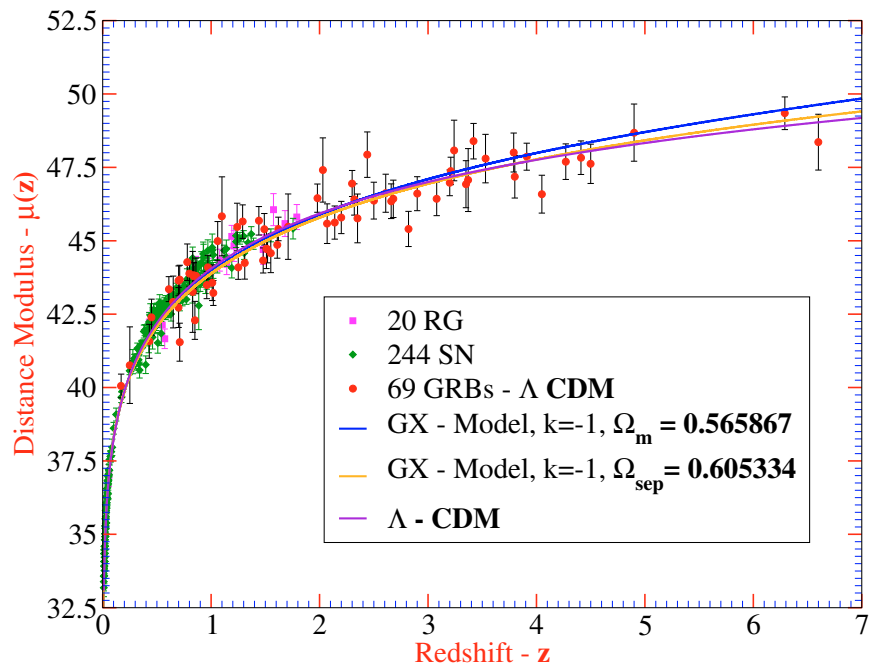

(b) $\mathrm{k}=-1$

Fig. 4. (Color online) a) The Hubble diagram for SNIa, radio-galaxies (RG), and GRBs calibrated both with GX model with $k=0, H_{0}=$ $70 \mathrm{~km} \mathrm{~s}^{-1} \mathrm{Mpc}^{-1}$, and $\Lambda$-CDM with $H_{0}=73 \mathrm{~km} \mathrm{~s}^{-1} \mathrm{Mpc}^{-1}$. SNIa: green points. RG: magenta points. GRBS: red points and black error bars. b) Hubble diagram for GX with $k=-1$ and $H_{0}=70 \mathrm{~km} \mathrm{~s}^{-1} \mathrm{Mpc}^{-1}$.

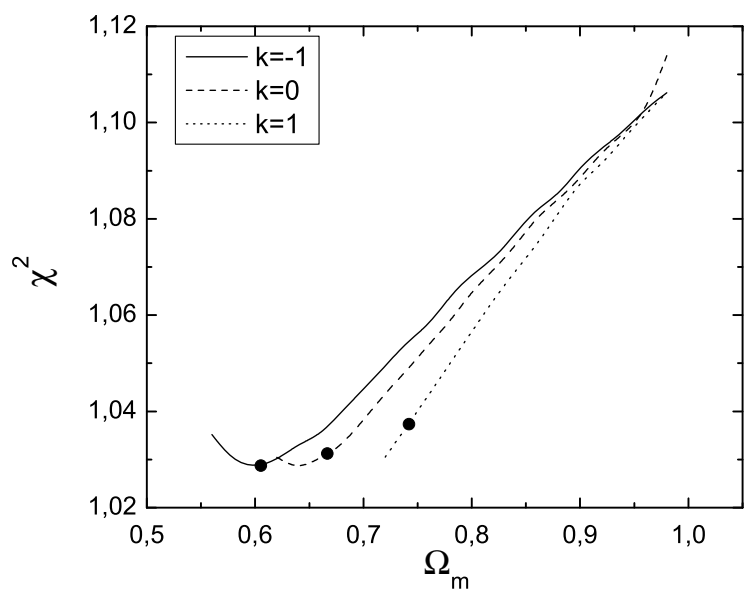

Fig. 5. (Color online) $\chi^{2}$ for the Hubble diagrams vs the $\Omega_{\mathrm{m}}$ for $k=$ $0,-1,1 \mathrm{GX}$-models. The fit points (circles) correspond to the separatrix.

For the same parameters, we have $\chi^{2} \approx 2$ with 264 DoF for SN (Khachatryan et al. 2007). The pair-best's fit values of the matter density parameter are close to the separatrix value for $k=0,-1,1$, which once again shows the important role of the separatrix in GX models. Indeed, we see that the separatrix fits the observational data best, with the shown values of the matter density parameter $\Omega_{\mathrm{m}}$ and the curvature $k$.

Concerning the $k=-1$ models, let us note that the ellipticity detected in the cosmic microwave background radiation temperature maps is characteristic of photon beam motion in hyperbolic spaces (Gurzadyan et al. 2005, 2007). Thus, it can act as a model-independent indication of the non precisely zero curvature of the Universe (Penrose 2005; Wiltshire 2007).

\subsection{GX compatibility with the 41 SWIFT satellite GRBs sample}

In this section we use the recently released SWIFT satellite, BAT instrument GRBs data (Sakamoto et al. 2007), namely,
Table 2. Confidence levels obtained after confronting both the supernovae and radio-galaxies datasets with the predictions of the GX cosmological model. The analysis were performed both separately and in combined tests for different values of $\Omega_{\mathrm{m}}$. All the cases analyzed correspond to $k=0$.

\begin{tabular}{lcc}
\hline \hline Sample & $\chi^{2}$ & $\Omega_{\mathrm{m}}$ \\
\hline SN244+RG20 & 1.23 & 0.508 \\
GRB69 & 1.04 & 0.64 \\
GRB41 & 1.41 & 0.66 \\
SN244+RG20+GRB69 & 1.58 & 0.66 \\
GRB69+GRB41 & 1.16 & 0.66 \\
GRBAll+SN244+RG20 & 1.56 & 0.66 \\
\hline
\end{tabular}

a sample of 41 GRBs known to have firmly determined redshifts (Rizzuto et al. 2007). We performed a similar procedure as Schaefer (2007), by constructing only three (3) luminosity indicator empirical relations from the SWIFT data. In particular by using the two variabilities defined by Rizzuto et al. (2007). Those empirical relations were constructed by taking into account the specific operational characteristics of the BAT instrument at the moment of analyzing the GRBs mask-tagged light curves $^{2}$. Those luminosity vs. luminosity indicator empirical

2 We proceeded as follows to obtain the Ghirlanda relation: we used the GRBs data collected within the SWIFT most energetic energy band; $1000 \mathrm{keV}$. We also assumed that the effective area of the BAT detector during those observations was $1000 \mathrm{~cm}^{2}$. In addition, we used several conversion factors because we did not have any explicit information about the $E_{\text {peak }}$ from the SWIFT data. We had only the energy flux data, $F_{\text {ene }}^{p}$ as tabulated in reference [arXiv:0707.4626v1]. Thus, we then "integrated" over $1 \mathrm{~s}$ and the quoted detector area (i.e., we implemented the conversion of) the flux to obtain the peak energy, and from there the Ghirlanda relation. All these informations were provided in all the figures we added to the present version. Besides, the GRBs luminosities were computed by following the procedure introduced by B. Schaefer in his paper on ApJ (2007). The main difference is that we did not get all the empirical relations by him utilized in that paper. We succeeded in constructing from the SWIFT GRBs data only those empirical relations presented in this last version. 
Table 3. Calibration relations for the GX cosmological model with parameters $k$ and $\Omega_{\mathrm{m}}$ as indicated.

\begin{tabular}{lccll}
\hline \hline $\begin{array}{l}\text { Luminosity relation } \\
k=0, \Omega_{\mathrm{m}}=0.6666\end{array}$ & $A$ & $B$ & $\sigma_{A}$ & $\sigma_{B}$ \\
\hline$\tau_{\text {lag }}-L$ & 52.2 & -1.08 & 0.0724 & 0.12 \\
$V-L$ & 52.4 & 1.76 & 0.102 & 0.247 \\
$E_{\text {peak }}-L$ & 52.2 & 1.71 & 0.0548 & 0.112 \\
$E_{\text {peak }}-E_{\gamma}$ & 50.5 & 1.62 & 0.0525 & 0.119 \\
$\tau_{\mathrm{RT}}-L$ & 52.5 & -1.25 & 0.0691 & 0.122 \\
\hline
\end{tabular}

$A$ and $B$ represent the linear regression intersept and slope as defined in the text, while $\sigma_{A}$ and $\sigma_{B}$ represent their respective errors, obtained through the linear regression after using the SLOPES method. Here $L$ is the luminosity, $\tau_{\text {lag }}$ is the time lag, $V$ is the variability, $E_{\text {peak }}$ is the peak (or maximum) energy, $E_{\gamma}$ is the corresponding energy in gammarays, and $\tau_{\mathrm{RT}}$ is the rise-time. All these variables are used in subsequent tables.

Table 4. Calibration relations for the GX cosmological model with parameters $k$ and $\Omega_{\mathrm{m}}$ as indicated.

\begin{tabular}{lccrr}
\hline \hline $\begin{array}{l}\text { Luminosity relation } \\
k=0, \Omega_{\mathrm{m}}=0.633\end{array}$ & $A$ & $B$ & $\sigma_{A}$ & $\sigma_{B}$ \\
\hline$\tau_{\text {lag }}-L$ & 52.2 & -1.09 & 0.0747 & 0.123 \\
$V-L$ & 52.4 & 1.71 & 0.101 & 0.243 \\
$E_{\text {peak }}-L$ & 52.1 & 1.67 & 0.0539 & 0.112 \\
$E_{\text {peak }}-E_{\gamma}$ & 50.4 & 1.6 & 0.0538 & 0.121 \\
$\tau_{\mathrm{RT}}-L$ & 52.4 & -1.22 & 0.0676 & 0.12 \\
\hline
\end{tabular}

Table 5. Calibration relations for the GX cosmological model with parameters $k$ and $\Omega_{\mathrm{m}}$ as indicated.

\begin{tabular}{lcrcc}
\hline \hline $\begin{array}{l}\text { Luminosity relation } \\
k=0, \Omega_{\mathrm{m}}=0.645\end{array}$ & $A$ & $B$ & $\sigma_{A}$ & $\sigma_{B}$ \\
\hline$\tau_{\text {lag }}-L$ & 52.2 & -1.09 & 0.0733 & 0.121 \\
$V-L$ & 52.5 & 1.78 & 0.103 & 0.248 \\
$E_{\text {peak }}-L$ & 52.2 & 1.73 & 0.0552 & 0.112 \\
$E_{\text {peak }}-E_{\gamma}$ & 50.5 & 1.63 & 0.0522 & 0.119 \\
$\tau_{\mathrm{RT}}-L$ & 52.5 & -1.26 & 0.07 & 0.123 \\
\hline
\end{tabular}

Table 6. Calibration relations for the GX cosmological model with parameters $k$ and $\Omega_{\mathrm{m}}$ as indicated.

\begin{tabular}{lcccc}
\hline \hline $\begin{array}{l}\text { Luminosity relation } \\
k=0, \Omega_{\mathrm{m}}=0.76\end{array}$ & $A$ & $B$ & $\sigma_{A}$ & $\sigma_{B}$ \\
\hline$\tau_{\text {lag }}-L$ & 52.1 & -1.05 & 0.0706 & 0.118 \\
$V-L$ & 52.4 & 1.71 & 0.101 & 0.243 \\
$E_{\text {peak }}-L$ & 52.1 & 1.67 & 0.0523 & 0.111 \\
$E_{\text {peak }}-E_{\gamma}$ & 50.4 & 1.6 & 0.0538 & 0.121 \\
$\tau_{\mathrm{RT}}-L$ & 52.4 & -1.22 & 0.0678 & 0.12 \\
\hline
\end{tabular}

relations are as follows (see a more detailed discussion, definitions and references in Rizzuto et al. 2007):

- Luminosity vs. Indicator: $V_{R}$ Variability - we built this relation based on data of $V_{\mathrm{R}}$ variability provided by Rizzuto et al. (2007) and SWIFT Flux data from Sakamoto et al. (2007). Rizzuto et al. (2007) proved that a Poissonian variance describes the statistical fluctuations of the GRBs lightcurves (see Apendix A in Rizzuto et al. 2007). The main reason is that the count rates that were used in the analysis already had their background substracted.

- Luminosity vs. Indicator: peak luminosity - this relation was constructed for each GRB as given in Table 1 of Rizzuto et al. (2007), which was obtained after extracting the mask-tagged light curve by using a binning time of $50 \mathrm{~ms}$ in the energy
Table 7. Calibration relations for the GX cosmological model with parameters $k$ and $\Omega_{\mathrm{m}}$ as indicated.

\begin{tabular}{lcrrr}
\hline \hline $\begin{array}{l}\text { Luminosity relation } \\
k=0, \Omega_{\text {sep }}=2 / 3\end{array}$ & $A$ & $B$ & $\sigma_{A}$ & $\sigma_{B}$ \\
\hline$\tau_{\text {lag }}-L$ & 52.2 & -1.08 & 0.0723 & 0.12 \\
$V-L$ & 52.4 & 1.76 & 0.102 & 0.247 \\
$E_{\text {peak }}-L$ & 52.2 & 1.71 & 0.0548 & 0.112 \\
$E_{\text {peak }}-E_{\gamma}$ & 50.5 & 1.62 & 0.0525 & 0.119 \\
$\tau_{\mathrm{RT}}-L$ & 52.5 & -1.25 & 0.0691 & 0.122 \\
\hline
\end{tabular}

Table 8. Calibration relations for the GX cosmological model with parameters $k$ and $\Omega_{\mathrm{m}}$ as indicated.

\begin{tabular}{lcccc}
\hline \hline $\begin{array}{l}\text { Luminosity relation } \\
k=-1, \Omega_{\mathrm{m}}=0.566\end{array}$ & $A$ & $B$ & $\sigma_{A}$ & $\sigma_{B}$ \\
\hline$\tau_{\text {lag }}-L$ & 52.3 & -1.11 & 0.0755 & 0.123 \\
$V-L$ & 52.5 & 1.82 & 0.104 & 0.25 \\
$E_{\text {peak }}-L$ & 52.3 & 1.77 & 0.0562 & 0.112 \\
$E_{\text {peak }}-E_{\gamma}$ & 50.6 & 1.66 & 0.0516 & 0.125 \\
$\tau_{\mathrm{RT}}-L$ & 52.6 & -1.29 & 0.0724 & 0.125 \\
\hline
\end{tabular}

Table 9. Calibration relations for the GX cosmological model with parameters $k$ and $\Omega_{\mathrm{m}}$ as indicated.

\begin{tabular}{lccll}
\hline \hline $\begin{array}{l}\text { Luminosity relation } \\
k=-1, \Omega_{\mathrm{m}}=0.578\end{array}$ & $A$ & $B$ & $\sigma_{A}$ & $\sigma_{B}$ \\
\hline$\tau_{\text {lag }}-L$ & 52.3 & -1.1 & 0.0746 & 0.122 \\
$V-L$ & 52.5 & 1.81 & 0.103 & 0.249 \\
$E_{\text {peak }}-L$ & 52.2 & 1.75 & 0.0558 & 0.112 \\
$E_{\text {peak }}-E_{\gamma}$ & 50.6 & 1.65 & 0.0521 & 0.121 \\
$\tau_{\mathrm{RT}}-L$ & 52.6 & -1.28 & 0.0713 & 0.124 \\
\hline
\end{tabular}

Table 10. Calibration relations for the GX cosmological model with parameters $k$ and $\Omega_{\mathrm{m}}$ as indicated.

\begin{tabular}{lcccc}
\hline \hline $\begin{array}{l}\text { Luminosity relation } \\
k=-1, \Omega_{\mathrm{m}}=0.605\end{array}$ & $A$ & $B$ & $\sigma_{A}$ & $\sigma_{B}$ \\
\hline$\tau_{\text {lag }}-L$ & 52.3 & -1.09 & 0.0732 & 0.121 \\
$V-L$ & 52.5 & 1.78 & 0.103 & 0.248 \\
$E_{\text {peak }}-L$ & 52.2 & 1.73 & 0.0551 & 0.112 \\
$E_{\text {peak }}-E_{\gamma}$ & 50.5 & 1.63 & 0.0522 & 0.119 \\
$\tau_{\mathrm{RT}}-L$ & 52.5 & -1.26 & 0.0698 & 0.123 \\
\hline
\end{tabular}

Table 11. Calibration relations of the SWIFT satellite GRBs data for the GX cosmological model with parameters $k$ and $\Omega_{\mathrm{m}}$ as indicated.

\begin{tabular}{lccccc}
\hline \hline $\begin{array}{l}\text { Luminosity relation } \\
k=0, \Omega_{\mathrm{m}}=0.666\end{array}$ & $A$ & $B$ & $\sigma_{A}$ & $\sigma_{B}$ & $\sigma_{\text {sys }}$ \\
\hline$V-L\left(V_{r}=0.05\right)$ & 49.902 & 1.98 & 0.487 & 0.448 & 0.729 \\
$V-L\left(V_{r}=0.02\right)$ & 49.902 & 1.98 & 0.487 & 0.966 & 0.966 \\
$V-L\left(V_{\mathrm{lp}}=0.05\right)$ & 52.744 & 1.634 & 0.222 & 0.3 & 0.3 \\
$V-L\left(V_{\mathrm{lp}}=0.005\right)$ & 51.11 & 1.634 & 0.443 & 0.352 & 0.352 \\
$E_{\text {peak }}-L($ Band $=1 \mathrm{MeV})$ & 52.5 & 1.961 & 0.19 & 0.805 & 0.805 \\
\hline
\end{tabular}

band [15-350] KeV (redshift-corrected). The $1 \mathrm{~s}$ time interval with the highest total counts was found, and assumed as the time interval corresponding to $1 \mathrm{~s}$ peak count rate in the subsequent analysis.

- Luminosity vs. Indicator: $V_{\mathrm{LP}}$ Variability - the same procedure as above, but notice that it was supposed that no extra-Poissonian variance had to be substracted from the already mask-tagged light curves. 


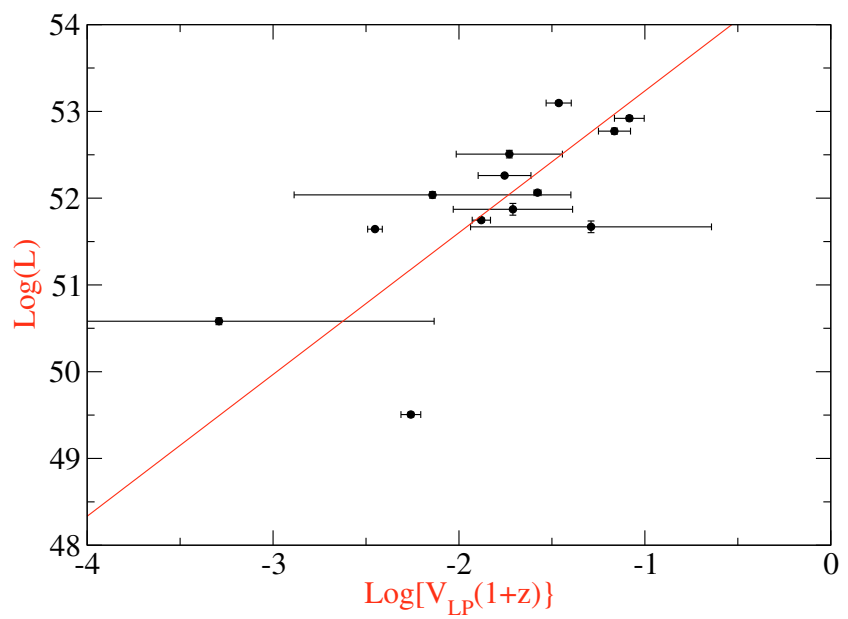

(a) Variability $V_{\mathrm{LP}}$ vs. $L$

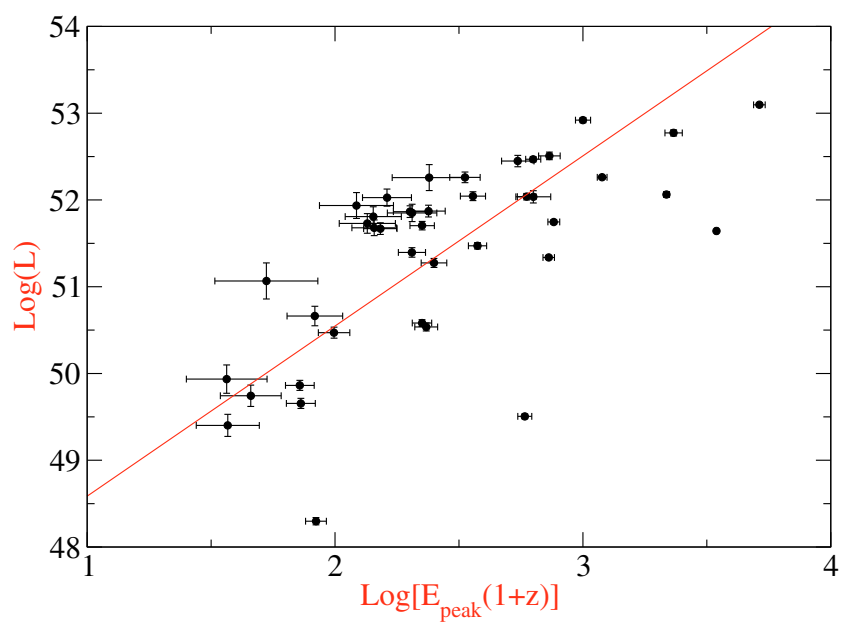

(c) $E_{\text {peak }}$ vs. $L$

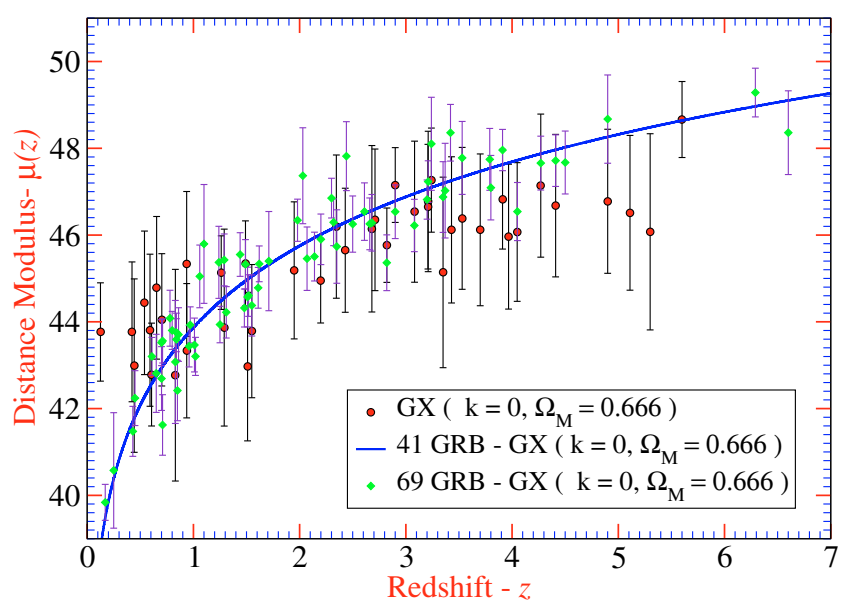

(e) $\mathrm{HD}$ for $V_{\mathrm{R}}, V_{\mathrm{LP}}$ with equal normalization constant 0.05

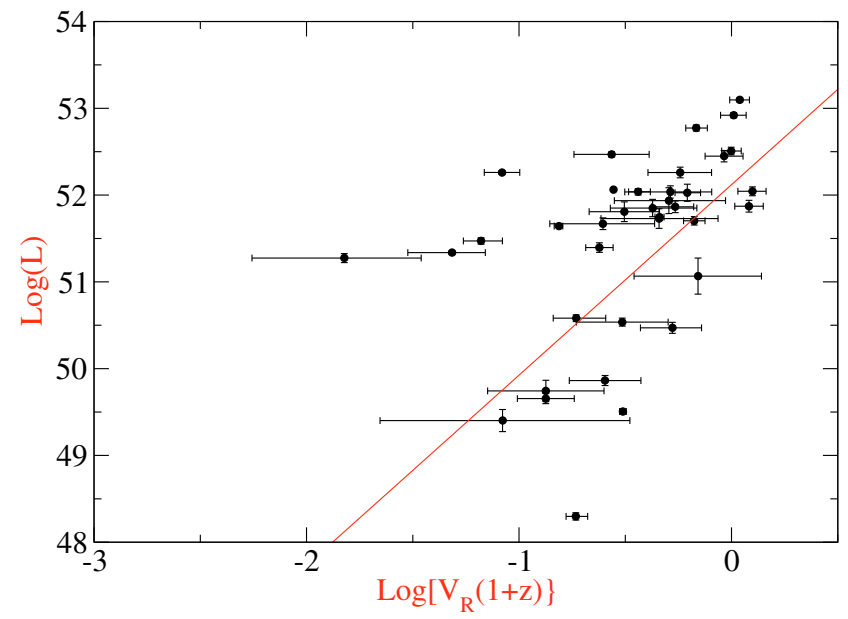

(b) Variability $V_{\mathrm{R}}$ vs. $L$

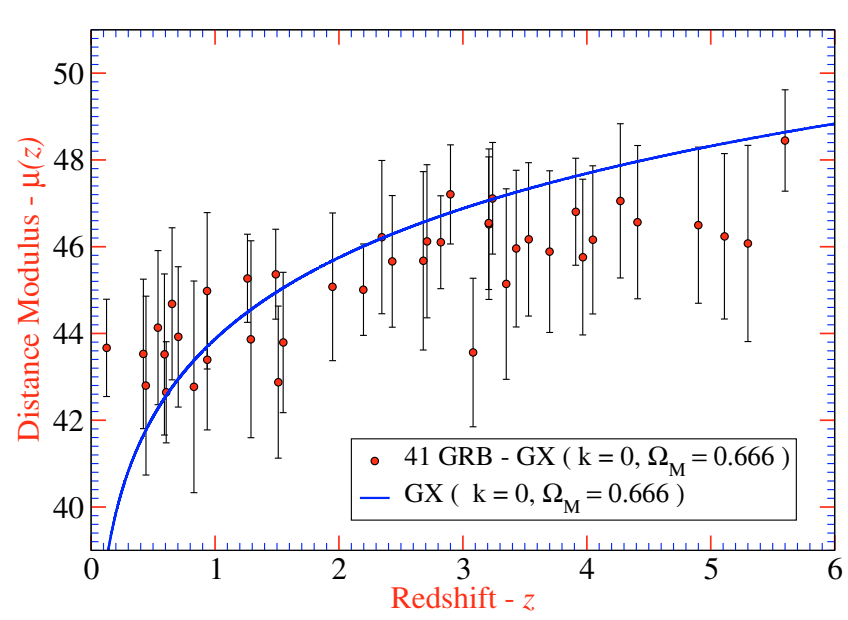

(d) Hubble diagram

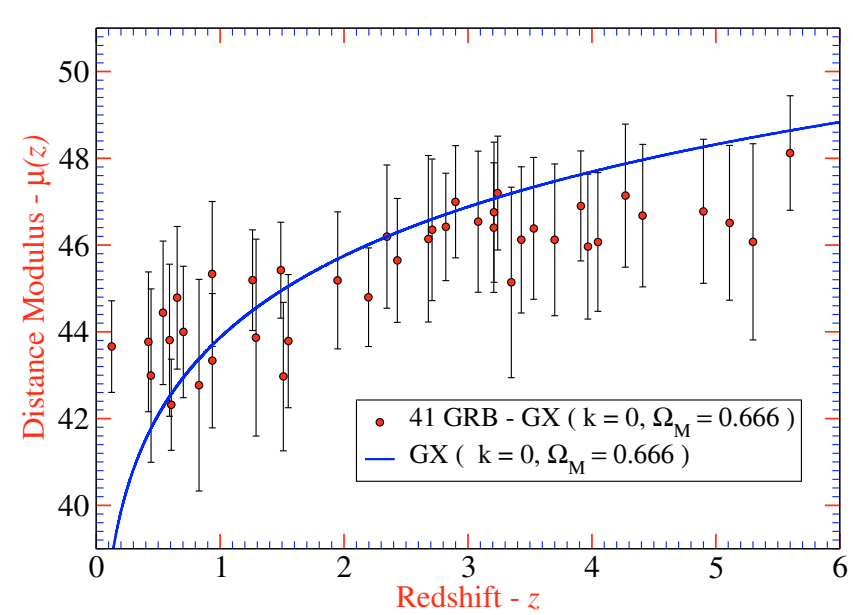

(f) $\mathrm{HD}$ for $V_{\mathrm{R}}, V_{\mathrm{LP}}$ with different normalization constant

Fig. 6. (Color online) Results of the calibration procedure for the SWIFT satellite 41 GRBs with redshift firmly determined (Rizzuto et al. 2007). Notice that all relations were corrected to the restframe of the GRB and also by using the luminosity best-fit line obtained from the nonlinear regression method. a) Variability $V_{\mathrm{LP}}$ for $13 \mathrm{GRBs}$ vs. isotropic luminosity. b) Variability $V_{\mathrm{R}}$ for 37 GRBs vs. isotropic luminosity. c) $E_{\text {peak }}$ values for 41 GRBs vs. peak isotropic luminosity. d) Hubble diagram (HD) from above relations with $V_{\mathrm{R}}=V_{\mathrm{LP}}$ normalization constant 0.02 . e) HD combining $41 \mathrm{SWIFT}$ and 69 Schaefer's GRBs samples, using $V_{\mathrm{R}}, V_{\mathrm{LP}}$ variabilities with equal normalization constant 0.05 . f) $V_{\mathrm{R}}, V_{\mathrm{LP}}$ variabilities with different normalization constant. 
Our results for the SWIFT GRBs data analysis are presented in Fig. 6, where the three empirical relations (6(a) $V_{\mathrm{LP}}$ vs. $L$, 6(b) $V_{\mathrm{R}}$ vs. $L, 6(\mathrm{c}) E_{\text {peak }}$ vs. $L$ ) described above are plotted. Besides, Figs. 6d-6f present three Hubble diagrams obtained by varying the normalization constant in the variability relations. Notice that Fig. 6e combines the SWIFT and Schaefer 69 GRBs data. These figures summarize the whole process of testing the consistency of the GX model (for several values of the parameters $k$ and $\Omega_{\mathrm{m}}$ ) with SWIFT GRBs data, as shown in Tables 3-11. The attentive reader should bear in mind that only three of those calibration processes are illustrated in Fig. 6. Figures $6 \mathrm{~d}-6 \mathrm{f}$ also reveal the sensitivity of the calibration procedure for these definitions of the GRBs variability on the normalization constant. The combined HD in Fig. 6e still appears to be consistent with the GX cosmological model, but it is also clear that the SWIFT GRBs data distort the better delineate HD of the Schaefer sample of 69 GRBs. Several values of the normalization constant were used in an attempt to understand the sensitivity of the resulting HD to such a parameter (see Figs. 6d, 6f). Also the systematic error is provided. This suggests that more data of high redshift GRBs detected by SWIFT would be needed to clarify the viability of using them as insight into GRBs precision cosmology. In this respect, perhaps the use of the SWIFT 77 GRBs sample with measured redshifts collected by Butler et al. (2007) may be of some help. That sample upon arrival of other new datasets will be used in future studies.

\section{Conclusion}

We have used the approach by Schaefer (2007) to obtain the GRB Hubble diagram for the cosmological GX models with dark energy. We have shown their fit to the observational data of GRBs with redshift up to $z \sim 6$ and obtained the best-fit values for the parameter $\Omega_{\mathrm{m}}$ defining the models (Table 2). For instance, the best-fits for the sample of $69 \mathrm{GRBs}$ correspond to $\Omega_{\mathrm{m}}=0.64 \pm 0.005$ and for $\mathrm{SN}+\mathrm{RG} \Omega_{\mathrm{m}}=0.508 \pm 0.004$.

In the context of the degeneracy between the cosmological parameters, it is remarkable that the GX-models predict the value of dark energy which is the same as the current one estimated from SN1a data.

The key issue in such an analysis is to what extent the GRBs can be used as standard candles, i.e. in revealing the genuine scatter in the empirical relations used for obtaining the Hubble diagram. Given the lack of understanding of the physics in those relations, handling the new data (Sato et al. 2007; Sakamoto et al. 2007; Rizzuto et al. 2007) must take a particular care. For example, Campana et al. (2007) claim weakening of the Ghirlanda relation ( $\chi^{2}$ up to 2 or 3 ) using a sample of 5 bursts observed by SWIFT. Even assuming that this result is correct, it will influence our evaluations negligibly $\left(\chi^{2}=1.040\right.$ instead of 1.037). However, Ghirlanda et al. (2007) reconsider the same SWIFT sample and show that such empirical relation survives practically unmodified. Cabrera et al. (2007) also report the correspondence of a sample of SWIFT data with those of other satellites and, hence, the absence of outliers.
In summary, the preliminary character of testing cosmological models via the GRB Hubble diagram indeed remains until we understand of the nature of the used empirical relations and availability of more observational data.

Acknowledgements. We thank the referee for many helpful comments. H.J.M.C. is fellow of the Fundação de Amparo à Pesquisa do Estado de Rio de Janeiro, FAPERJ, Brazil. The last three authors are partially supported by INTAS.

\section{References}

Astier, P., Guy, J., Regnault, N., et al. 2006, A\&A, 447, 31

Bloom, J. S., Frail, D. A., \& Kulkarni, S. R. 2003, ApJ, 594, 674

Butler, N. R., et al. 2007, ApJ, accepted [arXiv:0706.1275v3]

Cabrera, J. I., Firmani, C., Avila-Reese, V., et al. 2007, MNRAS, 382, 342

Campana, S., Tagliaferri, G., Malesani, D., et al. 2007, A\&A, 464, L25

Daly, R. A., \& Djorgovski, S. G. 2005 [arXiv: astro-ph/0512576]

Dai, Z. G., Liang, E. W., \& Xu, D. 2004, ApJ, 12, L101

Djorgovski, S. G., \& Gurzadyan, V. G. 2007, Nucl. Phys. B, 173, 6

Firmani, C., Avila-Reese, V., Ghisellini, G., \& Ghirlanda, G. 2006, MNRAS,

$$
\text { 372, L28 }
$$

Friedman, A. S., \& Bloom, J. S. 2005, ApJ, 627, 1

Ghirlanda, G., Ghisellini, G., \& Lazzati, D. 2004, ApJ, 616, 331

Ghirlanda, G., Nava, L., Ghisellini, G., \& Firmani, C. 2007 [arXiv: 0704. 0234]

Guerra, E. J., Daly, R. A., \& Wan, L. 2000, ApJ, 544, 659

Gurzadyan, V. G., \& Xue, S.-S. 2002, in From Integrable Models to Gauge Theories, 177 (World Scientific)

Gurzadyan, V. G., \& Xue, S.-S. 2003, Mod. Phys. Lett. A, 18, 561

Gurzadyan, V. G., de Bernardis, P., de Troia, G., et al. 2005, Mod. Phys. Lett. A, 20,813

Gurzadyan, V. G., Bianco, C. L., \& Kashin, A. L., et al. 2007, Phys. Lett. A, 363, 121

Isobe, T., Feigelson, E. D., Akritas, M. G., \& Babu, G. J. 1990, ApJ, 364, 104 Khachatryan, H. G. 2007, Mod. Phys. Lett., A22, 333

Khachatryan, H. G., Vereshchagin, G. V., \& Yegorian, G. 2007, Il Nuovo Cimento B, 122, 197

Liang, E. W., \& Zhang, B. 2005, ApJ, 633, 611

Liang, E. W., \& Zhang, B. 2006, MNRAS, 369, L37

Mosquera Cuesta, H. J., et al. 2006a [arXiv: astro-ph/0609262]

Mosquera Cuesta, H. J., et al. 2006b [arXiv: astro-ph/0610796]

Mosquera Cuesta, H. J., Dumet, H., \& Furlanetto, C. 2008, JCAP, 0807:004

Peebles, P. J. E. 1993, Principles of Physical Cosmology (Princeton Univ. Press)

Penrose, R. 2005, The Road to Reality, \#28.10, Jonathan Cape, London Perlmutter, S., Aldering, G., della Valle, M., et al. 1998, Nature, 391, 51 Perlmutter, S., Aldering, G., Goldhaber, G., et al. 1999, ApJ, 517, 565 Riess, A. G., Filippenko, A. V., Challis, P., et al. 1998, AJ, 116, 1009 Riess, A. G., Strolger, L.-G., Tonry, J., et al. 2004, ApJ, 607, 665

Riess, A. G., Strolger, L.-G., Casertano, S., et al. 2007, ApJ, 659, 98 Rizzuto, D., et al., 2007, MNRAS, 379, 619

Sakamoto, T., et al. 2007, ApJS, accepted [arXiv:0707.4626v1]

Sato, K., et al. 2007, ApJL, to appear [arXiv:0708.0263]

Schaefer, B. E. 2003, ApJ, 583, L67

Schaefer, B. E. 2007, ApJ, 660, 16

Vereshchagin, G. V. 2006 Mod. Phys. Lett., A21, 729

Vereshchagin, G. V., \& Yegorian, G. 2006a, Phys. Lett. B, 636, 150

Vereshchagin, G. V., \& Yegorian, G. 2006b, Class. Quantum Grav., 23, 5049

Vereshchagin, G. V., \& Yegorian, G. 2006c [arXiv:astro-ph/0604566]

Vereshchagin, G. V., \& Yegorian, G. 2008, Int. J. Mod. Phys., D17, 203

Wang, F. Y., \& Dai, Z. G. 2006, MNRAS, 368, 371

Wiltshire, D. 2007, New J. Phys., 9, 377

Xu, D., Dai, Z. G., \& Liang, E. W. 2005, ApJ, 633, 603

Zeldovich, Ya. B. 1967, JETP Lett., 6, 883

Zeldovich, Ya. B. 1968, Sov. Phys. Uspekhi, 95, 209 